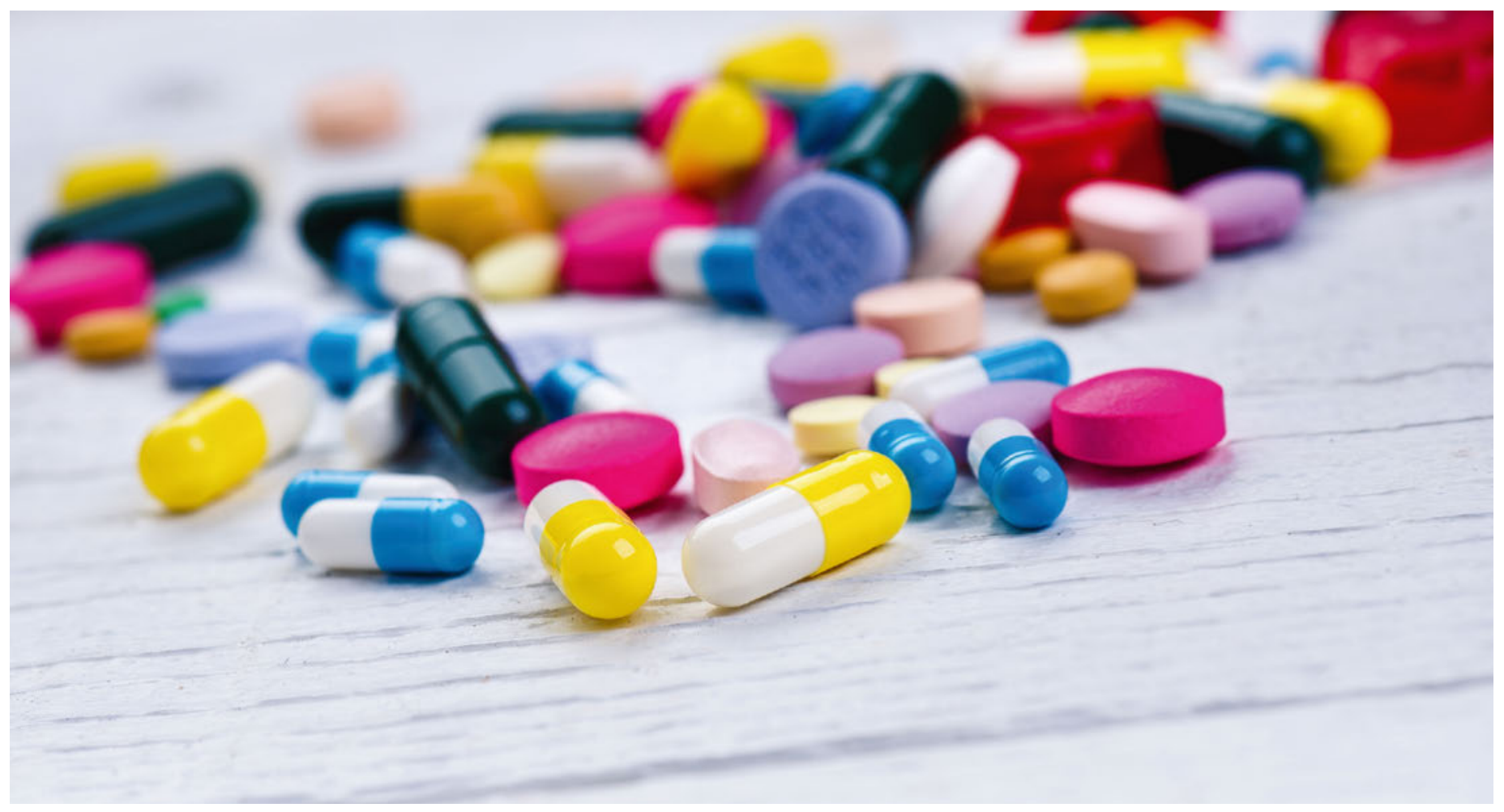

\title{
Ein hoffnungsvoller Pillen-Kick: Medication Reconciliation
}

\section{Bernhard Gurtner}

Dr. med., Mitglied FMH

Als Assistenzärzte an einem Zürcher Landspital haben wir vor bald 50 Jahren einige Medikationsregeln kennengelernt, die uns der medizinische Chefarzt als scharfzüngiger Appenzeller immer und immer wieder eingehämmert hat:

\section{Was der Bauer nicht kennt, frisst er nicht -} was der Arzt nicht kennt, verordnet er nicht Von allen Medikamenten, die ein Patient zu schlucken hatte, mussten wir die genaue Zusammensetzung, Pharmakokinetik, häufige Nebenwirkungen und Inter-

\section{Therapiepausen haben - soweit ich mich erinnere - nie zu einem ernsthaften Problem geführt.}

aktionen kennen. Auf Chefvisiten wurden wir darüber befragt, und wehe, wenn ... Die Angst vor Blamage bremste übereifrige Verordnungen und förderte pharmakologische Kenntnisse, die vor dem Staatsexamen in vielen Vorlesungen nur marginal vermittelt worden waren.

\section{Tauschhandel}

Muss unvermeidbar ein zusätzliches Medikament verordnet werden, soll jedesmal ein nicht mehr absolut notwendiges Präparat abgesetzt werden.

\section{Kleines Streichkonzert}

Oral verabreichte Medikamente auf zwölf Schluckeinheiten/Tag reduzieren.

\section{Grosses Streichkonzert \\ Alle Medikamente zwei Tage lang weglassen.}

Diese praktischen Empfehlungen haben sich ein ganzes Berufsleben lang nachhaltig bewährt. Sie konnten aber längst nicht alle Probleme lösen, die einem sicheren Medikamentenabgleich an den Schnittstellen der ambulanten und stationären Behandlung im Wege stehen. Wie gut behandelt fühlt sich ein Kranker, wenn er vor, während und nach einem Spitalaufenthalt ganz verschiedenartige Pillen, Kapseln und Tabletten schlucken oder weglassen muss? Das patientengefährdende und kostenverschlingende Chaos soll nun endlich dank 
einer interprofessionellen Verpflichtung mit vernetztem IT-Einsatz beseitigt werden [1-4]. Die Vereinbarung wurde von so vielen Gesundheitsbehörden, medizinischen, pflegerischen und pharmazeutischen Fachverbänden und Patientenschutzorganisationen unterzeichnet, dass Hoffnung besteht, der gute Wille der Spitzenkräfte sickere aus deren Sekretariaten und den Homepages baldmöglichst bis in die tägliche Praxis durch.

\section{Zu oft wird eine präklinische Polypharmazie unkritisch beibehalten.}

Einige frühere Konzepte sind in einem Arbeitsbehelf zur Reduktion der Polypharmazie [5] sehr gut zusammengefasst: PIM-Listen (Potentially Inappropriate Medication), MAI-Index (Medication Appropriateness Index) und der GPGP-Algorithmus von Garfinkel (Good Palliative Geriatric Practice) zur Reduktion der Medikation bei hochbetagten Patienten.

Therapiepausen haben - soweit ich mich erinnere - nie zu einem ernsthaften Problem geführt. Einige Patienten, die auf eigenen Wunsch und nach Besprechungen im Team und mit den Angehörigen sterben wollten, haben sich sogar nach Entzug aller gutgemeinten Pharmaka unerwartet erholt. Beschämend für uns Ärzte, die Interaktionen missachtet hatten. Garfinkel konnte nach Rücksprache mit allen Betroffenen bei 70 Patienten eines Geriatriezentrums in Israel 256 von 544 zuvor verschriebenen Arzneimitteln absetzen, wobei sich nur einmal der Verzicht auf Antikoagulation als fatal erwies. Hingegen habe sich der Allgemeinzustand nach Pillenreduktion bei 88 Prozent verbessert. Die eingeschränkte Medikation konnte während einer mehrjährigen Nachbeobachtungsphase beibehalten werden.

$\mathrm{Zu}$ oft wird eine präklinische Polypharmazie unkritisch beibehalten, um den Hausarzt nicht zu vertäuben, Spezialisten-Rezepte keinesfalls in Frage zu stellen und folgsame Patienten nicht zu verunsichern. Da auf manchen Visiten noch zusätzliche Medikamente hinzukommen, weil nette Assistenzärztinnen und weichherzige Kollegen auf jede Klage sofort mit einer Verordnung reagieren, wird den Pillenschluckern mehr geschadet als geholfen.

Etliche Patienten haben sich gegen die Medikamentenflut gewehrt, die Pillen heimlich in der Toilette weggespült oder aus dem Fenster geworfen. Das erklärte therapeutische Misserfolge und schützte vor Nebenwirkungen. Der Spitalgärtner hat die Pharma-Blindgänger jeweils eingesammelt, um seine Pflanzen vor Vergiftung zu bewahren.
Es gab aber auch Opposition gegen Streichkonzerte: Die medizinisch ausgebildete Tochter einer Patientin verlangte resolut, dass keine einzige Tablette aus dem täglichen Repertoire ihrer Mutter ohne telefonische Rückfrage - auch nachts! - gestrichen werde. Eine pharmazeutisch übersättigte alte Dame stimmte einer Therapiepause erst zu, als ihr eine permanente Sitzwache versprochen wurde, um notfalls einzugreifen. Selbst im kleinen Spital war es schwierig, eine für alle verbindliche Medikamentenliste zu vereinbaren und durchzusetzen. Neu hinzukommende Kaderärzte oder Fachspezialisten bestanden darauf, ihnen bekannte, im Haus aber nicht vorhandene Präparate auf die Liste zu setzen. Pharmavertreter brachten nicht nur erwünschte Neuentwicklungen, sondern versuchten auch, Medikamente zu platzieren, für welche kein Bedarf bestand. So bestellte ein Sparer der Verwaltung ohne Rücksprache einen grossen Posten Chloromycetin zum Schnäppchenpreis, weil man damit alle Lungenentzündungen billig behandeln könne.

Anekdotische Erinnerungen an gestern eignen sich nur bedingt als Hinweise für morgen, doch können ja nicht alle Eventualitäten des Medikamentenkonsums in Guidelines, Algorithmen und Apps eingebracht werden. Nun

\section{Etliche Patienten haben sich gegen}

die Medikamentenflut gewehrt, die Pillen heimlich in der Toilette weggespült oder aus dem Fenster geworfen.

sitzen alle im gleichen Boot, um einen baldigen Medikamentenabgleich mit kräftigen Ruderschlägen zu erzwingen. Bleibt zu hoffen, dass die am 4. November 2017 in Zürich spektakulär missglückte Weltpremiere einer Regatta mit 16er Booten kein böses Omen bedeutete. Immerhin ist das lange Schiff der Universität (mit Ärzten und Apothekern an Bord?) nicht auseinandergebrochen ...

\section{Bildnachweis}

(c) Tibor Ďuriš | Dreamstime.com

\section{Literatur}

1 Pilotprogramm "progress! Sichere Medikation an Schnittstellen», BAG, März 2015.

2 Charta «Sichere Medikation an Schnittstellen», Stiftung Patientensicherheit Schweiz, 31.10.2017.

3 Fishman L, Zimmermann C. Systematischer Medikationsabgleich im Praxistest, Schweiz Ärztezeitung. 2016;97(23):827-9.

4 Zimmermann C, Fishman L. Der systematische Medikationsabgleich in Schweizer Akutspitälern (zweijährige Pilotstudie in $8 \mathrm{Spi}$ tälern), Schweiz Ärztezeitung. 2017;98 (44):1451-3.

5 Arbeitsbehelf Reduktion der Polypharmazie der OÖGKK, Forum Gesundheit, Medieneigentümer und Herausgeber: OÖ Gebietskrankenkasse Linz mit Unterstützung der Österreichischen Pharmakologischen Gesellschaft und der Österreichischen Gesellschaft für Geriatrie und Gerontologie. Stand 10/2014. www.ooegkk. at/cdscontent/load?contentid=10008.603748\&version 